\title{
Dusuqing granules (DSQ) suppress inflammation in Klebsiella pneumonia rat via NF-kB/MAPK signaling
}

\author{
Xue Mei ${ }^{1,2,3 \dagger}$, Hao-Xun Wang ${ }^{4 \dagger}$, Jian-Sheng Li ${ }^{2,3^{*}}$, Xiao-Hui Liu' ${ }^{1}$, Xiao-Fan Lu ${ }^{2,3}$, Ya Li ${ }^{2,3,5}$, Wei-Yu Zhang ${ }^{2,3,5}$
} and Yan-Ge Tian ${ }^{2,3}$

\begin{abstract}
Background: Dusuqing granules (DSQ) have been used in the treatment of bacterial pneumonia clinically, with remarkable benefits. This study was initiated to explore the effects of DSQ on pulmonary inflammation by regulating nuclear factor (NF)-kB/mitogen-activated protein kinase (MAPK) signaling in bacterial pneumonia rats.

Methods: Rat model was duplicated with Klebsiella pneumonia by a one-time intratracheal injection. Rats were randomized into control, model, DSQ and levofloxacin (LVX) groups. After administrated with appropriate medicines for 7 days, lung tissues were harvested and prepared for pathological analysis, and interleukin (IL)-1, IL-6, monocyte chemotactic protein (MCP)-1and macrophage inflammatory protein (MIP)-2 detections. NF-KB mRNA was measured by real-time $\mathrm{qPCR}$, and the phosphorylation and total proteins of P38MAPK, JNK46/54, ERK42/44 were determined by Western blotting.
\end{abstract}

Results: Marked pathological impairments were observed in model rats, whereas were improved in DSQ group. The cytokines levels, NF-KB mRNA expression and the phosphorylation of P38MAPK, JNK46/54 and ERK42/44 proteins were significantly higher in model group, and were significantly depressed in DSQ group.

Conclusion: The protective effects of DSQ on Klebsiella pneumonia might be attributed to its inactivative effects of NF-KB/ MAPK pathway.

Keywords: Klebsiella pneumoniae pneumonia, Dusuqing granules, inflammation, NF-kB/MAPK pathway, Traditional Chinese medicine

\section{Background}

Klebsiellar pneumonia (KP), an important type of pneumonia typically in the form of bronchopneumonia and also bronchitis, is caused by Klebsiella pneumoniae ( $K$. pneumoniae), one of the most common Gram-negative pathogens and is becoming increasingly multidrug- resistant. KP is a major public health threat because of its severity, high incidence of complications, and elevated mortality, and its mortality rate was about $50 \%$ even

\footnotetext{
*Correspondence: li_js8@163.com

${ }^{\dagger}$ Equal contributors

${ }^{2}$ Henan Key Laboratory of Chinese Medicine for Respiratory Disease, Henan University of Chinese Medicine, Zhengzhou, Henan 450046, China

${ }^{3}$ Collaborative Innovation Center for Respiratory Disease Diagnosis and Treatment \& Chinese Medicine Development of Henan Province, Henan University of Chinese Medicine, Zhengzhou, Henan 450046, China Full list of author information is available at the end of the article
}

under antimicrobial therapy [1-4]. Nuclear factor (NF)$\mathrm{kB} /$ mitogen-activated protein kinase (MAPK) signaling pathway, important pathways involved in inflammation are closely related to the progress of KP. Signals are derived from pathogens or host cells, such as pathogenassociated molecular patterns (PMAP), danger-associated molecular patterns (DAMP). PAMP and DAMP are recognized by various pattern recognition receptors and ultimately cause the activations of MAPK (p38, ERK, JNK) and NF- $\kappa B$ signaling pathways. The pathways can initiate the gene transcriptions and promote expressions of various cytokines, such as interleukin (IL)-1, IL-6, tumor necrosis factor (TNF)- $\alpha$, monocyte chemotactic protein (MCP)-1, etc., and amplify the inflammatory response through a positive feedback cascade $[5,6]$. However, the 
cytokines production whose contribution to the host may be either protective or detrimental $[7,8]$.

Pneumonia belongs to the realm of syndrome of windheat invading lung in traditional Chinese medicine (TCM). Phlegm-heat syndrome always lasts through the whole process of the disease, and was often combined with qi and yin deficiency. Therefore, the method of heat-clearing, toxin-relieving and phlegm-resolving as well as replenishing qi and yin are the basis treatment of the disease. Based on this theory, our research group drew up the Dusuqing granules (DSQ), which has the effects of clearing heat, relieving toxin, resolving phlegm and replenishing qi and yin. In our previous randomized controlled trials, we found that DSQ can alleviate clinical symptoms and signs, improve quality of life of bacterial pneumonia patients $[9,10]$. In animal studies, it indicates that DSQ can lessen pulmonary inflammation and impairments, depress the levels of CRP and CR as well as proinflammatory cytokines $[11,12]$. In this study, we aimed to explore the effects of DSQ on pulmonary inflammation in bacterial pneumonia rats via regulating $\mathrm{NF}-\kappa \mathrm{B} / \mathrm{MAPK}$ signaling.

\section{Methods}

\section{Animals}

Twenty-eight male and 28 female Sprague-Dawley rats weighing 180 - 220 grams (g) were used in this study. All animals were obtained from Henan Laboratory Animal Center (Zhengzhou, Henan, China) and received humane care. The rats were randomly divided into control, model, DSQ, and levofloxacin (LVX) groups, 14 rats each group. The study protocol was approved by the Experimental Animal Care and Ethics committees in Henan University of Chinese Medicine (Zhengzhou, Henan, China). DSQ (batch: 130727) were purchased from the First Affiliated Hospital, Henan University of Chinese Medicine, Zhengzhou, Henan, China. The main components of the granule are as follows: Ren Shen (Radix ginseng) $10 \mathrm{~g}$, Mai Dong (Ophiopogon japonicus) $15 \mathrm{~g}$, Sheng Di Huang (radix rehmanniae recen)15g, Gua lou(trichosanthes kirilowii Maxim)20g, Yu Xing Cao(Houttuynia cordata)20g, Bai Tou Weng(the root of Chinese pulsatilla)10g. Levofloxacin tablets (batch: 20101001) were purchased from the Daiichi Pharmaceutical (Beijing) co., LTD.

\section{Klebsiella pneumoniae}

K. pneumoniae (Strain: 46114, National Center for Medical Culture Collections, Beijing, China) was cultured on nutrient agar plate and diluted to $2.4 \times 10^{8}$ colony forming unit $(\mathrm{CFU}) / \mathrm{mL}$ with normal saline before administered to animals.

\section{Model preparation}

$\mathrm{KP}$ rat models were prepared according to the reference [13]. After anesthetized with $10 \%$ chloral hydrate $(2.8$ $\mathrm{mg} / \mathrm{kg}$ ) on day 4 , K. pneumoniae solution $(0.1 \mathrm{~mL} / \mathrm{ani}$ mal) were given to the model rats with a one-time orotracheal intubation, while normal saline $(0.1 \mathrm{ml} / \mathrm{ani}$ mal) were given to the controls.

\section{Administrations}

After adaptive accommodation for 7 days, DSQ (2.8 $\left.\mathrm{mg} \cdot \mathrm{kg}^{-1} \cdot \mathrm{d}^{-1}\right)$ and LVX (31.64 $\left.\mathrm{mg} \cdot \mathrm{kg}^{-1} \cdot \mathrm{d}^{-1}\right)$ were given to rats in DSQ and LVX groups, respectively, 3 days before KP challenge, while the control and model rats were intragastrically administrated with normal saline $(2 \mathrm{ml} /$ animal). The equivalent doses of DSQ and LVX were calculated using the following formula: $D_{\text {rat }}=D_{\text {human }} \times$ $\left(\mathrm{K}_{\text {rat }} / \mathrm{K}_{\text {human }}\right) \times\left(\mathrm{W}_{\text {rat }} / \mathrm{W}_{\text {human }}\right)^{2 / 3}$ [14]. D: dose $(\mathrm{mg} / \mathrm{kg})$; $\mathrm{K}$ : body shape index; W: body weight. On the $4^{\text {th }}$ day, the models were made. From 5 to 7 day, the gavage solutions were carried out as the first three days. Body weights were measured on day 1 and 5 for the adjustments of the doses. All rats were sacrificed on day 8 .

\section{Cytological analysis in peripheral blood and bronchoalveolar lavage fluid}

The population of white blood cells and the percentage of polymorphonuclear neutrophils (PMN) in peripheral blood were measured by Hema-screen 18 (Italy), while they were counted in the bronchoalveolar lavage fluid (BALF) under an inverted microscopes (Olympus, Japan). Blood samples were sampled from caudal vein before sacrificed, and the BALFs were prepared with a three-time bronchoalveolar lavaging from the excised left lung lobes.

\section{Lung water content analysis}

Lung wet weights were measured and recorded after the lung lobes were excised, and dry weight were recorded after the lung tissues were totally dried in an air oven. Lung water content $(\mathrm{g} / 100 \mathrm{~g})=$ (wet weight - dry weight) $\times 100$ /wet weight.

\section{Morphology assessment}

For histological examination, after fixed in $4 \%$ paraformaldehyde for 72 hours, the lung tissues were paraffin embedded and sectioned into 4- $\mu \mathrm{m}$-thick slices, and stained with haematoxylin and eosin (H\&E) solutions. Images were captured at amplification of 200 under an Olympus PM-10 AD optical microscope and photographic system (Olympus, Tokyo, Japan). Lung injury, including alveolar congestion, hemorrhage, infiltration or aggregation of neutrophils in airspace or vessel wall, and thickness of alveolar wall, was blinded scored by a pathologist, according to a five-point scales used in 
previous study [15]: $0=$ minimal (little) damage, $1=$ mild damage, $2=$ moderate damage, $3=$ severe damage and 4 $=$ maximal damage. Total lung injury score was calculated as the sum of the above-mentioned four lesions.

\section{Enzyme linked immunosorbent assay}

After the excised lung tissues homogenized in $10 \times$ weight normal saline, the supernatant was separated and stored at $-80{ }^{\circ} \mathrm{C}$ for further analysis. The expressions of IL-1 $\beta$, IL-6, MCP-1 and MIP-2 in lung tissues were analyzed by enzyme linked immunosorbent assay (ELISA) according the introduction of the kits (Boster, Wuhan, China). The results were represented by $\mathrm{pg} / \mathrm{ml}$.

\section{Quantitative PCR analysis}

Total RNA was extracted by using TRIzol reagent (Invitrogen, US) according to the instruction and assessed by agarose gel electrophoresis and absorbance measurements at 260 and 280 nanometer $(\mathrm{nm})$ wavelength on Thermo BIONANO Ultramicro ultraviolet light spectrophotometer (Thermo Forma, MA, USA). Complementary DNA (cDNA) was synthesized with a SuperRT cDNA kit (CW Biotechnology, Beijing, China) according to the manufacturer's standard protocol, while PCR was performed by using Quantifast ${ }^{\circ} \mathrm{SYBR}^{\circ}$ Green PCR Kit (QIAGEN, Germany). The reaction systems were prepared following the instructions of the kits. The initial activation was at $95{ }^{\circ} \mathrm{C}$ for $15 \mathrm{~s}, 60^{\circ} \mathrm{C}$ for $1 \mathrm{~min}$ on an $\mathrm{ABI}$ 7500 Fast real time instrument (ABI, CA, USA). The primers of NF- $\mathrm{kB}$ and glyceraldehyde-3-phosphate dehydrogenase (GAPDH) were designed and synthesized by Generay Biotech Co. Ltd. (Shanghai, China). NF-кB: forward (5'-3'): ACG ATC TGT TTC CCC TCA TCT, reverse (5'-3'): TGG GTG CGT CTT AGT GGT ATC; GAPDH: forward (5'-3'): GGT GAA GGT CGG TGT GAA CG, reverse (5'-3'): CTC GCT CCT GGA AGA TGG TG.

\section{Western blotting analysis}

Lung tissues were homogenized in ice-cold lysis buffer containing $1 \mathrm{mM}$ phenylmethanesulfonyl fluoride (PMSF) and $1 \times$ protease inhibitor cocktail (Sigma-Aldrich, USA) for $40 \mathrm{~min}$, then centrifuged at 12,000 $\mathrm{g}$ for $20 \mathrm{~min}$. Protein concentrations were measured using the BCA assay. $30 \mu \mathrm{g}$ of total protein was sampled and separated in $10 \%$ sodium dodecyl sulfate/polyacrylamide gel electrophoresis (SDS-PAGE) and transferred to polyvinylidene difluoride (PVDF) membranes (Millipore, MA, USA). The membranes were then blocked with $5 \%$ fatfree milk in Tris-buffered saline and $0.5 \%$ Tween-20 (TBS-T) for $2 \mathrm{~h}$ and then incubated with corresponding primary antibodies overnight at $4{ }^{\circ} \mathrm{C}$. After washing with TBS- $T$, the membranes were incubated with horseradish peroxidase (HRP)-conjugated goat anti-rabbit antibody
(ZB2306) for $1 \mathrm{~h}$ at room temperature. Finally, signals were gained by using the enhanced chemiluminescence (ECL) kit (CW Biotechnology, Beijing, China) and were exposed by using a biological molecular imaging instrument (LAS4000mini, JAPAN). The immunoblots intensities were quantified using Quantity One software (Bio-Rad, CA, USA). The primary antibodies including P38 (D13E1), p-P38 (D3F9), ERK (137F5), P-ERK (D13.14.4E), JNK (56G8), p-JNK (98F2), $\beta$-Tublin (9F3) and GAPDH $(14 \mathrm{C} 10)$ were purchased from Cell Signaling Technology, Inc. (CST, MA, USA).

\section{Statistical analysis}

Statistical analyses were performed using SPSS Statistics 19.0 software (SPSS, IL, USA). One-Way ANOVA was applied for comparison among groups. All data were expressed as mean \pm standard deviation (SD). $P<0.05$ was set as the statistical significance.

\section{Results \\ Mortality}

On day 5 and 6, three rats died in model group and one in LVX group due to pulmonary infection and abscess, one rat died in DSQ group due to flatulence.

\section{Lung water content}

Lung water content of the rats was significantly higher in model group compared with control group $(P<0.01)$. Compared with model group, it was significantly lower in DSQ and LVX groups $(P<0.01)$. Especially, it was significantly lower in DSQ group than that in LVX group $(P<0.01)$ (Table 1).

\section{Histomorphology}

As shown in Fig. 1, the lung tissues in model group were found damaged and expressed alveolar congestion, hemorrhage, alveolar wall thickened, and inflammatory cells infiltration in the interstitium and alveolar spaces, and were reduced in DSQ and LVX groups. No apparent impairment was observed in control rats.

Lung injury score was significantly higher in model group compared with controls $(P<0.01)$, and it was significantly lower in DSQ and LVX groups $(P<0.01)$. There was no significance between DSQ and LVX group $(P>0.05)$ (Table 2).

Table 1 Lung Water Content in each group (mean \pm SD)

\begin{tabular}{lll}
\hline Group & $\mathrm{N}$ & Lung water content \\
\hline control & 14 & $62.30 \pm 1.44$ \\
model & 11 & $94.06 \pm 2.20^{* *}$ \\
DSQ & 13 & $75.86 \pm 1.11^{\# \# \mathbf{\Lambda}}$ \\
LVX & 13 & $80.80 \pm 0.70^{\# \#}$ \\
\hline Note: ${ }^{* *} P<0.01$ vs. control group; ${ }^{\# \#} P<0.01$ vs. model group; ${ }^{\mathbf{\Lambda}} P<0.01$ vs. LVX group
\end{tabular}




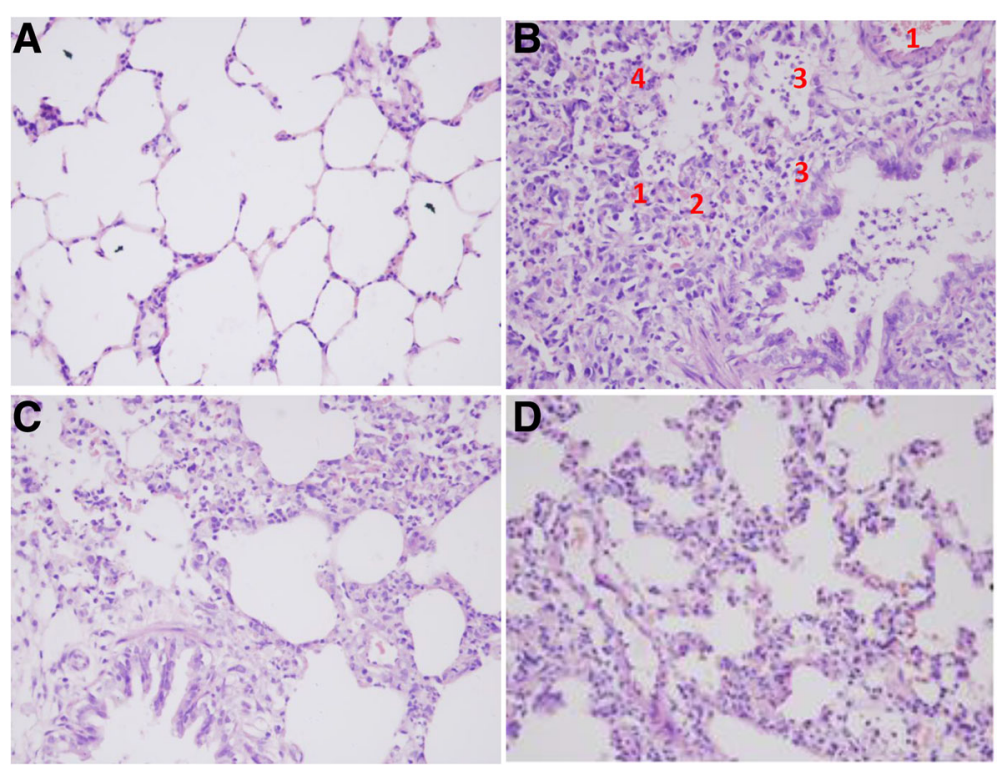

Fig. 1 Pulmonary histomorphology in each group (H\&E stained). (a) control group (b) model group (c) DSQ group; (d) LVX group. 1: alveolar congestion, 2: hemorrhage, 3: infiltration or aggregation of neutrophils, 4: thickness of alveolar wall (B)

\section{WBC population and PMN percent in peripheral blood}

WBC population and PMN percent increased significantly in peripheral blood in model group compared with control group $(P<0.01)$, and they were significantly lower in DSQ and LVX groups compared to model rats $(P<0.01)$. There was no significance between DSQ and LVX group $(P>0.05)$ (Table 3$)$.

\section{WBC and PMN populations in BALF}

The populations of WBC and PMN in BALF increased significantly in model group compared with control group $(P<0.01)$, and were decreased significantly in DSQ and LVX groups $(P<0.05)$. There was no significance between DSQ and LVX group $(P>0.05)$ (Table 4$)$.

\section{IL-1 $\beta$, IL-6, MCP-1 and MIP-2 in lung tissue}

As shown in Fig. 2, IL-1 $\beta$, IL-6, MCP-1 and MIP-2 were significantly higher in model group than control group $(P<0.01)$. Compared with model group, IL-1 $\beta$, IL-6, MCP-1 and MIP-2 decreased significantly in DSQ and

Table 2 Lung Injury Score (mean \pm SD)

\begin{tabular}{lll}
\hline Group & $\mathrm{N}$ & Score \\
\hline control & 14 & $1.50 \pm 0.84$ \\
model & 11 & $12.17 \pm 0.75^{* *}$ \\
DSQ & 13 & $8.17 \pm 0.75^{\# \#}$ \\
LVX & 13 & $8.33 \pm 0.82^{\# \#}$ \\
\hline Note: ${ }^{* *} P<0.01$ vs. control group; ${ }^{\# \#} P<0.01$ vs. model group &
\end{tabular}

LVX groups $(P<0.01)$. MCP-1 and MIP-2 were lower in DSQ group than LVX group $(P<0.05)$.

\section{Expression of NF-KB mRNA}

As shown in Fig. 3, Compared with control group, the expression of NF- $\mathrm{kB}$ mRNA in model group increased significantly $(P<0.01)$, while it was significantly lower in DSQ and LVX groups compared to model group $(P<0.05)$.

\section{P38MAPK, JNK54/46 and ERK1/2 protein and their phosphorylation in each group}

As shown in Fig. 4, Significant elevation of p- P38MAPK, $\mathrm{p}$-JNK and p-ERK1/2 levels was observed in model group compared with control group $(P<0.01)$, while the expressions of p- P38MAPK and p-JNK were significantly lower in DSQ and LVX groups than in model group $(P<0.05$, $P<0.01)$. The level of $\mathrm{p}-\mathrm{ERK} 1 / 2$ were lower in DSQ group than in model group $(P<0.05)$, while there was no difference in p-ERK1/2 level between LVX group and model group $(P>0.05)$.

Table 3 WBC population and PMN percent in peripheral blood in each group (mean $\pm \mathrm{SD}$ )

\begin{tabular}{lcll}
\hline Group & $N$ & WBC $\left(\times 10^{9} / \mathrm{L}\right)$ & $\mathrm{PMN}(\%)$ \\
\hline control & 14 & $6.38 \pm 1.23$ & $24.22 \pm 5.11$ \\
model & 11 & $13.52 \pm 2.86^{* *}$ & $56.24 \pm 6.37^{* *}$ \\
DSQ & 13 & $8.60 \pm 2.86^{\# \#}$ & $37.25 \pm 4.22^{\# \#}$ \\
LVX & 13 & $9.29 \pm 1.80^{\# \#}$ & $35.44 \pm 6.42^{\# \#}$ \\
\hline Note: ${ }^{* *} P<0.01$ vs. control group; ${ }^{\# \#} P<0.01$ vs. model group
\end{tabular}


Table 4 WBC and PMN populations in bronchoalveolar lavage fluid in each group (mean \pm SD)

\begin{tabular}{llll}
\hline Group & $\mathrm{N}$ & WBC $\left(\times 10^{6} / \mathrm{L}\right)$ & $\mathrm{PMN}\left(\times 10^{6} / \mathrm{L}\right)$ \\
\hline control & 14 & $17.14 \pm 2.29$ & $8.82 \pm 2.07$ \\
model & 11 & $24.45 \pm 4.71^{* *}$ & $16.64 \pm 2.75^{* *}$ \\
DSQ & 13 & $21.36 \pm 3.76^{\#}$ & $13.65 \pm 3.17^{\#}$ \\
LVX & 13 & $20.84 \pm 3.34^{\#}$ & $13.95 \pm 3.16^{\#}$ \\
\hline Note: ${ }^{* *} P<0.01$ vs. control group; ${ }^{\# \#} P<0.01$ vs. model group &
\end{tabular}

\section{Discussion}

The present study demonstrates that Dusuqing granules, an effective formula clinical used for the treatment of community-acquired pneumonia in the elderly play distinctly suppressant roles on inflammatory response in Klebsiella pneumonia rats induced by a one-time attack of $K$. pneumoniae infection via regulating NF- $\mathrm{B} / \mathrm{MAPK}$ pathway.

The pattern of phlegm-heat obstructing lungs is one of the most common syndromes in bacterial pneumonia, which happens in the elderly always with deficient vital root, qi-yin deficiency syndrome, or qiyin impaired by long-term phlegm-heat pathogens. DSQ is specific preparation for the syndromes of phlegm-heat obstructing lungs combined with qi-yin deficiency. It has been confirmed effective in alleviating systemic and pulmonary inflammation and impairments, improving clinical symptoms, and improving quality of life of community-acquired pneumonia patients in previous studies [9-12]. High component complexity of traditional Chinese herbal prescription has its unique advantages and characteristics of therapeutic effects via multi-level, multichannel and multi-target [16]. In this study, we aimed to reveal the further mechanism of DSQ in treating bacterial pneumonia via the regulation of $N F-k B /$ MAPK signaling.

Klebsiella pneumonia is one kind of most common lung infections. As one of phagocytes that constitute an integral component of innate immune defense, PMNs migrate to inflammation sites to eliminate pathogens, early in the acute inflammatory response to infection and injury [17]. Our data indicated that DSQ can significantly reduce the white blood cell population and PMN rate in peripheral blood. Additionally, DSQ can also alleviate pathological changes in the lungs, including reducing inflammatory cell infiltration in airspace and vessel walls, alleviating pulmonary congestion and hemorrhage, and decreasing alveolar wall thickening. These results are consistent with previous studies $[11,18]$.

$\mathrm{NF}-\kappa \mathrm{B}$ is the most important transcriptional regulator that regulate the inflammatory pathways. Activated $N F-\kappa B$ by a wide array of mediators can translocate to the nucleus and bind to the promoters of pro-inflammatory genes, leading to enhanced gene expression and amplification of the inflammatory response which lead to the inflammatory injury. Therefore, blocking NF- $\mathrm{kB}$ transcriptional activity may be an important target for treating inflammatory diseases [19-21]. In this study, NF-kB mRNA was observed significantly elevated in the lungs of $\mathrm{KP}$ rats, DSQ can suppress the expression of NF- $\mathrm{KB}$
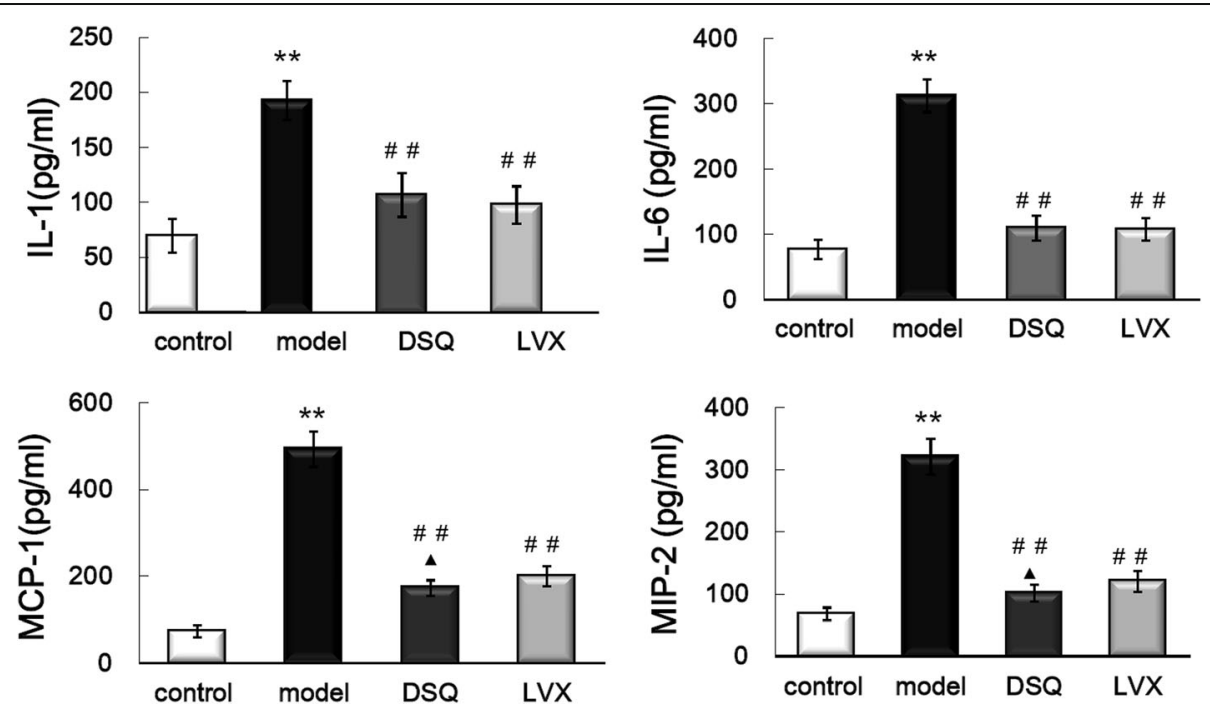

Fig. 2 Levels of IL-1ß, IL-6, MCP-1 and MIP-2 in the lungs in control, model, DSQ and LVX groups ${ }^{* *}<<0.01$ vs. control group; ${ }^{\# \#<0.01 ~ v s . ~ m o d e l ~}$ group; ${ }^{\wedge}<0.05$ vs. LVX group. Values are expressed as mean \pm S.D., one-way ANOVA 


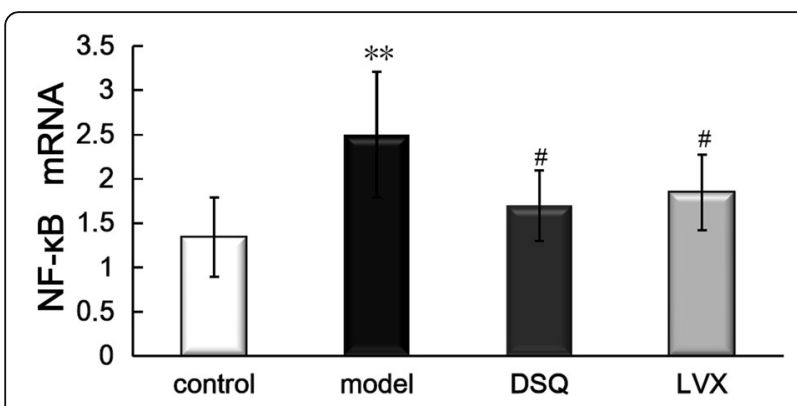

Fig. 3 Expressions of NF-kB mRNA in the lungs in control, model, Dusuqing and Levofioxacin ${ }^{* *} P<0.01$ vs. control group; ${ }^{\#} P<0.05$ vs. model group; Values are expressed as mean \pm S.D., one-way ANOVA

mRNA, and DSQ showed similar regulative effects with LVX.

The MAPK signaling pathway, classified into extracellular signal regulated kinases $1 / 2$, c-Jun $\mathrm{N}$ terminal kinase, and p38MAPK, is implicated in the release of immune-related cytotoxic factors and proinflammatory cytokines, and inhibitors of MAPK reduce pro-inflammatory protein levels [22, 23]. Data indicate that the activation of NF- $\mathrm{KB} / \mathrm{MAPK}$ signaling contributes to KP-induced cytokine/chemokine production in the lungs [24]. Decreasing of the phosphorylation level of NF- $\mathrm{kB}$ and P38 can downregulate the productions of cytokines, and minimize the injuries in lung tissue [25]. In this study, we found that the phosphorylation levels of P38 MAPK, JNK and ERK1/2 increased significantly in the lungs of KP rats. DSQ can suppress the phosphorylation of MAPK. Our data indicate DSQ can decrease the productions of IL-1 $\beta$, IL-6, MCP-1 and MIP-2 which increased significantly in the lungs of KP rats by inhibiting NF- $\mathrm{KB}$ and MAPK.

\section{Conclusion}

In conclusion, DSQ have significant effects on reducing pulmonary pathological impairments and inflammatory responses, and the inhibiting effects of NF- $\mathrm{BB}$ and MAPK signaling of DSQ might be involved in its underlying mechanisms, and these data provide new evidence for the clinical application of Traditional Chinese Medicine.
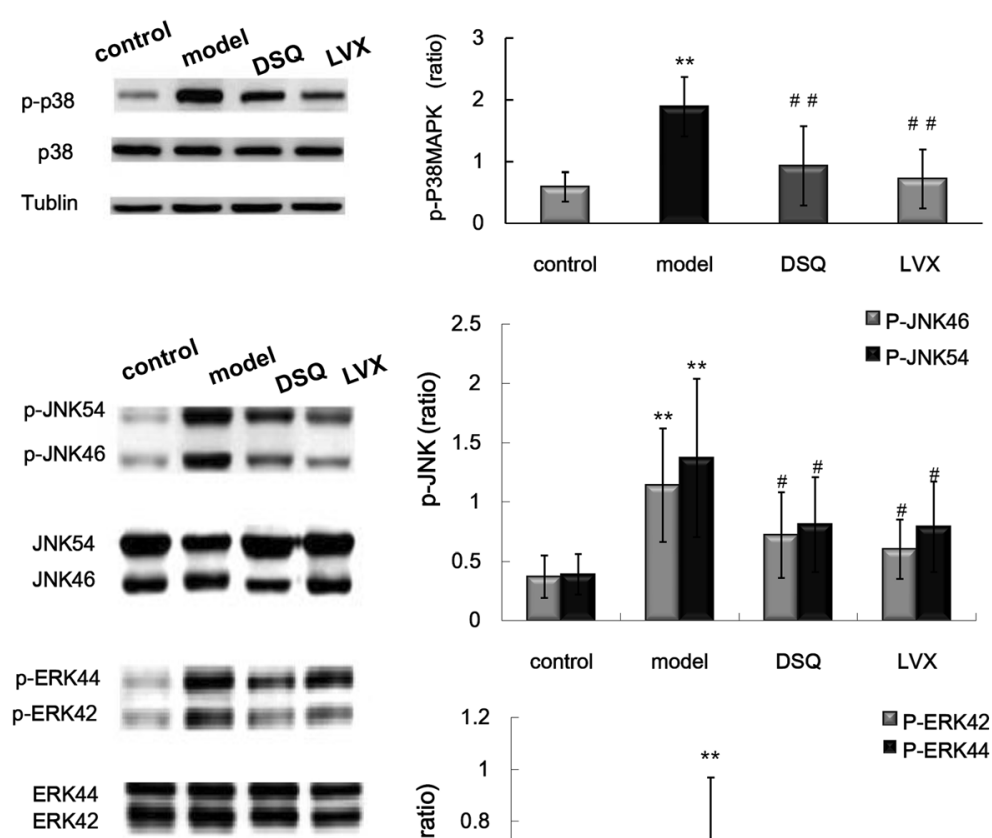

GADPH

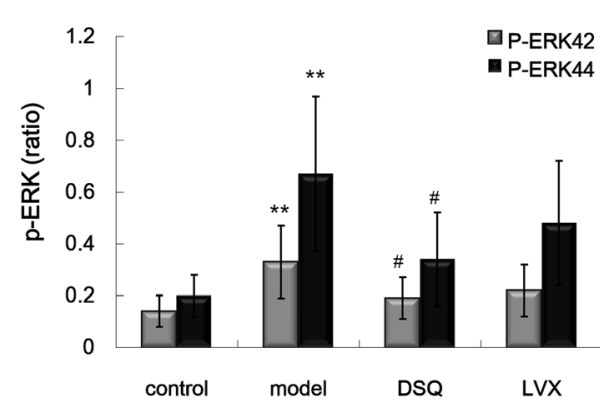

Fig. 4 Phosphorylation of P38MAPK, JNK and ERK1/2 in the lungs in control, model, DSQ and LVX groups ${ }^{* *} P<0.01$ vs. control group; ${ }^{*} P<0.05$, \#\# $P<0.01$ vs. model group. Values are expressed as mean \pm S.D., one-way ANOVA 


\section{Abbreviations}

DSQ: Dusuqing; GAPDH: Glyceraldehyde-3-phosphate dehydrogenase; IL: Interleukin; KP: Klebsiellar pneumonia; LVX: Levofloxacin; MAPK: Mitogen-activated protein kinase; MCP-1: Monocyte chemotactic proteinand-1; MIP-2: Macrophage inflammatory protein-2; NF-KB: Nuclear factor-kB

\section{Acknowledgments}

None.

\section{Funding}

This research was funded by Natural Science Foundation of China, with the grant number 81202658

\section{Availability of data and materials}

The datasets supporting the conclusions of this article are included within the article.

\section{Authors' contributions}

MX, LS and WHX contributed to the study design. MX also contributed to data analysis, manuscript drafting and process control. WHX also contributed data analysis and manuscript drafting. LY and TYG contributed to protein analysis and data collection. LXH and TYG contributed to RNA analysis and data collection.MX and LXH contributed to cytokines analysis. LXF and ZWY contributed to animal experiment. All authors had read and approved the final manuscripts.

\section{Competing interests}

The authors declare that they have no competing interests.

\section{Consent for publication}

Not applicable.

\section{Ethics approval}

The study protocol was approved by the Experimental Animal Care and Ethics committees in Henan University of Chinese Medicine (Zhengzhou, Henan, China).

\section{Publisher's Note}

Springer Nature remains neutral with regard to jurisdictional claims in published maps and institutional affiliations.

\section{Author details}

'Basic Medicine College, Henan University of Chinese Medicine, Zhengzhou, Henan 450046, China. ${ }^{2}$ Henan Key Laboratory of Chinese Medicine for Respiratory Disease, Henan University of Chinese Medicine, Zhengzhou, Henan 450046, China. ${ }^{3}$ Collaborative Innovation Center for Respiratory Disease Diagnosis and Treatment \& Chinese Medicine Development of Henan Province, Henan University of Chinese Medicine, Zhengzhou, Henan 450046, China. ${ }^{4}$ The Second Affiliated Hospital, Zhengzhou University, Zhengzhou, Henan 450014, China. ${ }^{5}$ The First Affiliated Hospital, Henan University of Chinese Medicine, Zhengzhou, Henan 450000, China.

\section{Received: 28 September 2016 Accepted: 8 April 2017}

\section{Published online: 17 April 2017}

\section{References}

1. Li X, Zhou X, Ye Y, Li Y, Li J, Privratsky B, Wu E, Gao H, Huang C, Wu M. Lyn regulates inflammatory responses in Klebsiella pneumoniae infection via the p38/NF-kB pathway. Eur J Immunol. 2014;44:763-73.

2. Holmes RB. Friedlander's pneumonia. Am J Roentgenol Radium Therapy, Nucl Med. 1956;75:728-47.

3. Korvick AJ, Hackett AK, Yu LV, Muder RR. Klebsiella pneumoniae in the modern era: Clinicoradiographic correlations. South Med J. 1991;84:200-4.

4. Chuang TY, Lin CJ, Chi CL, Liu AY, Lee SW, Lin TL, Wang JT, Hsueh PR. Rapidly fatal bacteremic pneumonia caused by Klebsiella pneumoniae with K1 hypermucoviscosity phenotype in a previously healthy young man receiving levofloxacin treatment. J Microbiol Immunol Infect. 2009;42:439-41.

5. Kang H, Bang T-S, Lee J-W, Lew J-H, Eom SH, Lee K, Choi H-Y. Protective effect of the methanol extract from Cryptotaenia japonica Hassk Against lipopolysaccharide-induced inflammation in vitro and in vivo. BMC Complement Altern Med. 2012;12:199.

6. Li Y, Li J-s, Li W-W, Li S-y, Tian Y-g, Lu X-f, Jiang S-I, Wang Y. Long-term effects of three Tiao-Bu Fei-Shen therapies on NF-kB/TGF- $31 /$ smad2 signaling in rats with chronic obstructive pulmonary disease. BMC Complement Altern Med. 2014;14:-140.

7. Rivas-Santiago B, Juárez E. Toll-like receptor in lung response to pathogens. Rev Investig Clin. 2007;59:481-8.

8. Knapp S, Schultz MJ, van der Poll T. Pneumonia models and innate immunity to respiratory bacterial pathogens. Shock. 2005;24:12-8.

9. Li JS, Cheng L, Ma LJ, Kong GL, Li SY, Wang K, Chen XL, Wu JZ. Clinical Observation on DSQ in Treating Senile Bacterium Pneumonia. Chinese Journal of Integrated Traditional And Western Medicine. 2002;22:505-7.

10. Zhou SZ, Li SY. Effect of DSQ Granule in Treatment of 19 Patients of Senile Bacterium Pneumonia. Henan Traditional Chinese Medicine. 2007:27:51.

11. Zhang YX, Li JS, Zhou HY, Qiao CX, Yu HB. Effect of Dusuqing on the Inflammatory Factors in the Lung Tissue of Bacterial Pneumonia and Heatphelgm Combining with Disease and Syndrome. Liaoning Journal of Traditional Chinese Medicine. 2009;36:1616-8.

12. Li YK, Li JS, Zhang J. Effect of DUSUQING on the AP in Aged Rats with Bacterium Pneumonia. China Journal of Modern Medicine. 2002;12:21-3.

13. Li JS, Yu CC, Li SY, Zou YL, Li DW. Effect of Dusuqing on the inflammatory cytokine in aged rats' heart injury of Klebsiella pneumonia. Chinese Journal of Integrated Traditional \& Western Medicine in Intensive \& Critical Care. 2008;15:74-7.

14. Huang JH, Huang XH, Cheng ZY, Zheng QS, Sun RY. Dose conversion among different animals and healthy volunteers in pharmacological study. Zhongguo Lin Chuang Yao Li Xue Yu Zhi Liao Xue. 2004;9:1069-72.

15. Liu D, Zeng BX, Shang Y. Decreased expression of peroxisome proliferatoractivated receptor gamma in endotoxin-induced acute lung injury. Physiol Res. 2006:55:291-9.

16. ShiKai YAN, Jing ZHAO, Sheng-Shan DOU, Peng JIANG, Run-Hui LIU, WeiDong ZHANG. Methodology of Modernization Research in Traditional Chinese Medicine Based on Systems Biology and Network Biology. Chin J Nat Med. 2009:7:249-59.

17. Nathan C. Neutrophils and immunity: Challenges and opportunities. Nat Rev Immunol. 2006:6:173-82.

18. Li JS, Yu SC, Li SY, Zou YL, Li DW. Effect of Dusuqing on the inflammatory cytokine in aged rats'heart Injury of Klebsiella pneumoniae. Chin J TCM WM Crit Care. 2008;15(2):74-7.

19. Sun YN, Li W, Yan XT, Yang SY, Song SB, Kim YH. Phenolic components from the stem of Acanthopanax koreanum and their inhibitory effects on NF-kappa B. Biosci Biotechnol Biochem. 2014;78:374-7.

20. Liu M-W, Mei-xian S, Zhang W, Wang Y-q, Chen M, Wang L, Qian C-y. Protective effect of Xuebijing injection on paraquat-induced pulmonary injury via down-regulating the expression of p38 MAPK in rats. BMC Complement Altern Med. 2014:14, 498.

21. Xu X, Liu N, Zhang Y-X, Cao J, Wu D, Peng $Q$, Wang HB, Sun WC. The Protective Effects of HJB-1, a Derivative of 17-Hydroxy-Jolkinolide B, on LPSInduced Acute Distress Respiratory Syndrome Mice. Molecules. 2016;21(1) doi:10.3390/molecules21010077.

22. Nadeem A, Siddiqui N, Alharbi NO, Alharbi MM, Imam F. Acute glutathione depletion leads to enhancement of airway reactivity and inflammation via p38MAPK-iNOS pathway in allergic mice. Int Immunopharmacol. 2014;22:222-9.

23. Guo R, Wu K, Chen J, Mo L, Hua X, Zheng D, Chen P, Chen G, Xu W, Feng J. Exogenous hydrogen sulfide protects against doxorubicin-induced inflammation and cytotoxicity by inhibiting p38MAPK/NFKB pathway in H9c2 cardiac cells. Cell Physiol Biochem. 2013;32:1668-80.

24. Cai S, Batra S, Shen L, Wakamatsu N, Jeyaseelan S. Both TRIF- and MyD88Dependent Signaling Contribute to Host Defense against Pulmonary Klebsiella Infection. J Immunol. 2009;183(10):6629-38.

25. Li X, Zhou X, Li Y, Li J, Privratsky B, Ye Y, Wu E, Gao H, Huang C, Wu M. Lyn cooperating with lipid rafts regulates inflammatory responses in Klebsiella pneumoniae infection through the p38/NF-kB pathway. Eur J Immunol. 2014:44:763-73. 